\title{
CLINICA-ESCOLA DE PSICOLOGIA: PRINCIPAIS PSICOPATOLOGIAS EM PACIENTES DO GÊNERO FEMININO NA FAIXA ETÁRIA DE 25 A 35 ANOS.
}

\author{
Zilda Rodrigues, Daniel Azevedo do Nascimento, Carol Milagros Kohatsu Casazola \\ Universidade do Oeste Paulista - UNOESTE, Curso de Psicologia, Presidente Prudente, SP. E-mail: zilda@unoeste.br
}

\begin{abstract}
RESUMO
A Clínica-escola é um espaço para a pratica clínica dos discentes e de pesquisa para docentes e discentes em psicologia. $O$ presente estudo tem como objetivo discutir as principais psicopatologias encontradas em pacientes do gênero feminino no serviço de triagem da clínicaescola de psicologia em uma universidade no interior do Estado de São Paulo - Brasil, nos anos compreendidos entre 2005 a 2014, pertencentes a faixa etária de 25 a 35 anos. A abordagem utilizada foi a Quantitativo-descritiva, a metodologia envolveu a coleta de dados de triagens armazenadas no Sistema Informatizado da Clínica-Escola. Os resultados revelaram a prevalência de Episódios Depressivos (40,14\%), Outros Transtornos de Ansiedade (11,97\%) e Transtornos Fóbicos Ansiosos (11,27\%), caracterizando a depressão e os transtornos de ansiedade como as principais psicopatologias no gênero feminino nessa faixa etária.

Palavras-chave: Clinica-Escola; Triagem; Psicopatologia; Gênero feminino; Faixa etária.

\section{CLINIC-SCHOOL OF PSYCHOLOGY: MAIN PSYCHOPATHOLOGIES IN PATIENTS OF THE FEMALE GENDER IN THE AGE GROUP 25 TO 35 YEARS OLD}

\begin{abstract}
The Clinic-school is a space for clinical practice of students, as well as the practice of research for students and teachers. This study aimed to discuss the main psychopathologies found in female patients in screening service of clinical-school of psychology at a university in the State of São Paulo - Brazil, in the years among 2005 to 2014, belonging the age group 25-35 years old. With Quantitative and descriptive approach, the methodology involved collecting screening data stored in the computerized system of the Clinic-School. Results showed the prevalence of depressive episodes (40.14\%), Other Anxiety Disorders (11.97\%) and Phobic Disorders Anxious (11.27\%), characterizing depression and anxiety disorders as the main psychopathologies in the genre women in this age group.
\end{abstract}

Keywords: Clinic-School; Screening; Psychopathology; Gender Female, Age group. 
INTRODUÇÃO

No Brasil desde o ano 1962, a formação da pratica clínica de psicologia ocorre por meio das chamadas Clínicas-Escolas, as quais devem oferecer serviços obrigatórios pela legislação que dispõe sobre o curso de formação em Psicologia. A profissão é regulamentada no Brasil pela Lei № 4.119.1962. Assim, a Clínica-escola transita em dois âmbitos fundamentais, a prestação de serviço à comunidade e o exercício supervisionado da pratica clínica (CAMPEZATTO, NUNES. 2007).

Para Silvares (2006) o Brasil é o único país na América do Sul que conta com o serviço da Clínica-Escola dentro das Faculdades de Psicologia, o qual é caracterizado como o espaço em que o futuro psicólogo desenvolve nos últimos anos de graduação a parte prática de sua formação clínica. Outra função importante da clínica-escola é o espaço para desenvolver pesquisa clínica, pois ela propicia melhor entendimento de como ocorrem mudanças nas pessoas, contribuindo também para a formação de futuros pesquisadores. Dentro da clínica-escola os estagiários geralmente iniciam suas práticas com o estágio em triagem ou também chamada entrevista diagnóstica ou entrevista inicial, que para Rocha (2011) tem a função de especificar a demanda do cliente encaminhando a um serviço especifico, uma outra função da triagem é o levantamento da hipótese diagnóstica para isso utiliza-se a Classificação Internacional de Doenças - CID 10, que através de uma linguagem padronizada de uma série de doenças, possibilita comparar sintomas e queixas.

$\mathrm{Na}$ clínica-escola é frequente a queixa de psicopatologias, presentes no CID-10 como Classificação de Transtornos mentais e de comportamento. A psicopatologia não pretende o julgamento moral, busca apenas observar, identificar e compreender os diversos elementos da doença psíquica. Este estudo inicia-se pela observação cuidadosa de suas manifestações, fazendo articulação dialeticamente com a ordenação dos fenômenos, o que representa que, para observar, também é preciso produzir, definir, classificar, interpretar e ordenar o observado de forma lógica. (DALGALARRONDO, 2008).

No Brasil tem se realizado algumas pesquisas em clínica-escola, sendo elas de descrição de clientela, triagem ou serviços diferenciados (AMARAL, 2012). E o perfil que se destaca nos atendimentos é a prevalência de atendimento do gênero feminino na idade adulta, confirmando praticamente o mesmo nas diversas pesquisas (MARAVIESKI, SERRALTA, 2011). Pesquisas não só nas clínicas-escolas mais também em estudos epidemiológicos vêm afirmando a prevalência do gênero feminino nos serviços de saúde. Ora, por gênero não devemos identificar somente o sexo como é comum acontecer, mais a identidade de si próprio a partir do sexo biológico, como diz Kaplan, Sadock e Grebb (1997, p, 642) "o conjunto determinado de atitudes, comportamento e outros atributos geralmente associados com a masculinidade ou feminilidade". 
Para Rubin (1975 apud ARAÚJO, MATIOLLI, 2004) sexo e gênero se distingue à medida que um caracteriza diferenças biológicas e o outro a construção social e cultural sobre as diferenças sexual. Caracterizando gênero como o resultado de um processo das relações sociais que restringe aos homens características somente masculinas e nas mulheres características somente femininas.

Scott (1990; 1994 apud Siqueira 2008) a respeito do gênero discorda de Rubin (1975) afirmando que este debate o conceito somente sobre a lente do parentesco. A visão Scott (1990) sobre o gênero ergue-se "socialmente" como já afirmado por Rubin e outros, no entanto, amplia-se para além do parentesco, transitando entre as relações políticas, econômica, de educação e mercado de trabalho. A autora acima ainda afirma:"[...] o gênero é uma forma primeira de dar significados as relações de poder". O que deixa claro uma diferença entre os gêneros onde um está subordinado ao outro.

Na visão de Amaral (2012) o número de pesquisas na clínica-escola ainda é insuficiente comparado com o número de cursos de psicologia no pais. Face a essa constatação e a importância da clínica-escola como um lócus de aprendizagem, a demanda social por serviços psicológicos, considerando também a insuficiência de pesquisas, entende-se que o presente trabalho apresenta relevância social e acadêmica.

A pesquisa visa discutir e caracterizar as psicopatologias mais frequentes nas pacientes do gênero feminino de 25 a 35 anos de idade que buscaram atendimento na clínica escola de psicologia, nos anos compreendidos entre 2005 a 2014.

Metodologia

Para alcançar seu objetivo este estudo foi inscrito na Coordenadoria Central de Pesquisa (CCPq/UNOESTE) sob protocolo (2190), não necessitando da autorização do Comitê de Ética (CEP/UNOESTE). O aporte metodológico, apoiou-se na pesquisa Documental, quantitativo-descritiva; procedimento utilizado para compreensão e análise de documentos de variados tipos (SÁ-SILVA, ALMEIDA, GUINDANI. 2009).

A coleta ocorreu a partir de um levantamento dos dados armazenados no Sistema Informatizado da Clínica Escola pertencente a uma universidade do interior do estado de São Paulo Brasil, provenientes do Serviço de triagem e não houve contato com informações pessoais das pacientes. A faixa etária escolhida pareceu aos pesquisadores como a melhor para ser estudada, pois, conforme apresenta Cordioli (2009), os cuidados com os filhos pequenos, as solicitações profissionais, os cuidados com a casa e muitas outras tarefas, colaboram para o desgaste psíquico.

Realizada a coleta, as principais psicopatologias foram selecionadas por critério de incidência de casos, partindo de uma análise de frequência. Com a ajuda de planilhas eletrônicas (EXCEL) os 
dados das subcategorias do CID-10, foram agrupadas em uma tabela em forma decrescente em suas respectivas categorias. Para a identificação das principais psicopatologias adotou-se uma incidência maior que $10 \%$ de casos.

\section{RESULTADOS}

O período pesquisado contabilizou 206 triagens com mulheres na faixa etária determinada pela pesquisa, dentre essas triagens, apenas 142 casos informam psicopatologias, as outras 64 classificações referem-se a problemas de ciclo vital, crise existencial e outros, classificados pelo CID10 nas categorias: Z60, Z61, Z62, Z63, Z64, Z72, Z73, G40, R45, Y05, Z34, esses dados não foram tratados, pois, o objeto da presente pesquisa são as psicopatologias.

$\mathrm{Na}$ tabela encontra-se as 142 triagens agrupadas e suas respectivas psicopatologias, catalogadas pela Classificação Internacional de Doenças (CID-10) como Transtornos Mentais e Comportamentais.

Tabela. Distribuição de Psicopatologias

\begin{tabular}{lcc} 
Categoria & $F$ & $\%$ \\
\hline F.32 Episódio Depressivo & 57 & 40,14 \\
\hline F.41 Outros Transtornos de Ansiedade & 17 & 11,97 \\
\hline F.40 Transtorno Fóbico Ansioso & 16 & 11,27 \\
\hline F.60 Transtorno Especifico de Personalidade & 14 & 9,86 \\
\hline F.33 Transtorno Depressivo Recorrente & 9 & 6,34 \\
\hline F.43 Reação a estresse grave e transtornos de ajustamento & 7 & 4,93 \\
\hline F.45 Transtornos Somatoformes & 4 & 2,82 \\
\hline F.81 Transtornos Específicos do Desenvolvimento das Habilidades & 4 & 2,82 \\
Escolares & 2 & 1,41 \\
\hline F.63 Transtornos de Hábitos e Impulsos & 2 & 1,41 \\
\hline F.31 Transtorno Afetivo Bipolar & 2 & 1,41 \\
\hline F.70 Retardo Mental Leve & 1 & 0,70 \\
\hline F.34 Transtornos Persistentes do Humor (Afetivos) & 1 & 0,70 \\
\hline F.42 Transtorno Obsessivo Compulsivo & 1 & 0,70 \\
\hline F.66 Transtornos Psicológicos e de Comportamento Associados & & \\
ao Desenvolvimento e Orientação Sexuais & 1 & 0,70 \\
\hline F.10 Transtornos Mentais e de Comportamento decorrentes do & 1 & 1 \\
Uso de Álcool & 1 & 0,70 \\
\hline F.20 Esquizofrenia & 1 & 0,70 \\
\hline F.25 Transtornos Esquizoafetivos & 1 & 0,70 \\
\hline F.72 Retardo Mental Grave & 100,00 \\
\hline F.50 Transtornos Alimentares & 140 \\
\hline & & 1 \\
\hline
\end{tabular}


Considerando o total de psicopatologias é possível observar a predominância dos casos de depressão e transtornos com sintomatologia predominante de ansiedade; em vista da frequência de registros de Episódios Depressivo (40,14\%), Outros Transtornos de Ansiedade (11,97\%), Transtorno Fóbico Ansioso (11,27\%). A partir desses dados é possível afirmar que o maior número de queixa presente no serviço de triagem da clínica-escola procede de transtornos depressivos e ansiosos.

\section{DISCUSSÃO}

O estudo feito comparado a pesquisas já realizadas anteriormente, apesar da distinção dos métodos e técnicas, revelam semelhanças nos resultados. Por exemplo, o que diz Campezato e Nunes (2007) em sua pesquisa, as mulheres se queixam mais de problemas relacionados a depressão, ansiedade. Romaro e Oliveira (2008) estudando a queixa de adultos separados relata maior número de queixas de ansiedade, insegurança, medo $(13,5 \%)$ e depressão $(11 \%)$. Com o objetivo de encontrar o perfil clinico e sociodemográfico de uma clínica-escola Maravieski e Serralta (2011) puderam verificar nas mulheres a prevalência das queixas de sintomas depressivos (26\%) e ansiedade $(13,7)$.

Conforme os resultados deste estudo, os Episódios Depressivos (40,14\%), seguido por Outros Transtornos de Ansiedade (11,97\%) bem como Transtornos Fóbicos Ansiosos $(11,27 \%)$, são as psicopatologias com maior número de casos no serviço de triagem, em mulheres de 25 a 35 anos na clínica-escola. A partir dessas informações se observa que neste grupo em particular mais de $63 \%$ das psicopatologias triadas trata-se de depressão ou transtorno de ansiedade. Sendo possível afirmar que para cada caso de transtorno de ansiedade em mulheres no serviço de clínica-escola existem dois casos de depressão.

As pesquisas que tratam de epidemiologia têm demonstrando uma maior incidência e prevalência de transtornos mentais e comportamentais no gênero feminino. Conforme observado, reconhece nas mulheres taxas superiores aos dos homens em transtornos de ansiedade e, do humor. Podendo em diversos casos o transtorno de ansiedade estar associado a depressão. (ANDRADE, VIANA, SILVEIRA, 2006; APOSTOLO, 2011).

Em estudo para estimar a prevalência de transtornos psiquiátricos na cidade de São Paulo, Andrade e Viana (2012) identificaram nas mulheres a prevalência de transtornos de ansiedade e de humor. Molina et. al. (2012) ao pesquisar a prevalência da depressão em usuários de unidades de atenção primaria, constatou que nas mulheres é encontrado "quase o dobro de depressão" que nos homens. Sendo que o maior número de mulheres com caso de depressão se encontram na faixa etária de 20 a 59 anos, ou seja, por toda a fase adulta as mulheres superam os homens em casos de 
depressão. Apoiando-se nesses e em outros estudos é possível afirmar que as mulheres são mais vulneráveis a casos de depressão e de ansiedade. Visto que para cada homem com depressão é registrado dois ou mais casos em mulheres em idade produtiva (FURUYA et al. 2013; TENG, HUMES, DEMETRIO, 2005; JUSTO, CALIL, 2006).

Segundo demonstra Kinrys e Wygant (2005), os transtornos de ansiedade são mais diagnosticado nas mulheres. Nos casos de fobia social, transtorno de ansiedade generalizada e transtorno de pânico, as mulheres tem apresentado duas vezes mais casos do que homens, além de ser possível encontrar mais comorbidades associadas, elevando a gravidade dos sintomas. Informações confirmadas por Andrade, Viana e Silveira (2006), que supõem essa prevalência de gênero em fatores biológicos e ambientais, "além de fatores de maior aceitação cultural do medo e comportamento de esquiva em mulheres e diferentes padrões adaptativos, os homens tendem a utilizar substancias, como a nicotina [...] mascarando a sintomatologia" (p.47). Mas no que diz respeito a mascarar sintomatologias, de acordo com Castro et al. (2008) as mulheres apresentam mais associação entre ansiedade e depressão a gravidade de dependência a nicotina, do que homens. Que conforme estudo realizado leva a crer que as mulheres também fazem uso do cigarro como "automedicação". E assim como os homens, podem mascarar uma serie de sintomas.

A respeito dos fatores que influenciam e contribuem para a predominância da depressão sobre o gênero feminino; o biológico, psíquico e social atuam no desenvolvimento, manutenção e remissão. Segundo Andrade, Viana e Silveira (2006), os hormônios femininos, em particular o estrógeno, atua na modulação do humor, o que, poderia descrever a maior ocorrência dos transtornos de ansiedade e do humor nas mulheres. A época da menarca desempenha um papel importante na explicação desta diferença, já que a partir da adolescência $5 \%$ da população feminina demonstra uma prevalência maior que os homens. Demetrio (2000) afirma que "A desproporção mulher/homem ocorre no período reprodutivo, sendo a prevalência semelhante antes da puberdade e também após alguns anos da transição menopausa" (Apud TENG, HUMES, DEMETRIO, 2005; BAPTISTA, BAPTISTA, OLIVEIRA 1999).

No que diz respeito a discussão, os transtornos discutidos neste trabalho e muitos outros, afetam tanto as mulheres quanto os homens. No entanto, dentro de todo o período da idade adulta, verifica-se a prevalência de ansiedade e depressão na mulher. Quer dizer, na fase de produção e desenvolvimento profissional, econômico e de constituição familiar, as mulheres sofrem mais com problemas de humor e ansiedade. Do ponto de vista das relações de gênero, analisando a partir do contexto social e histórico. A referência patriarcal é extremamente atuante, uma vez que cria e normatiza as relações sociais, dando dinâmica ao poder que impera nas relações de gênero. 
Poder esse que inscreve as mulheres em uma atuação ou papel determinado, tanto no espaço público como no privado (LAGO, 2006). Onde ao longo do tempo naturalizou atividades mais realizadas pelas mulheres, ao ponto dessas atividades se tornarem invisíveis, o que desvaloriza, exigindo o dobro e as vezes o triplo do trabalho, o que sobrecarrega e adoece muitas vezes as mulheres.

Conclusão

Este estudo aponta como resultado a prevalência de depressão e transtornos de ansiedade no gênero feminino, com idade de 25 a 35 anos. Informações essas confirmadas pelos diversos estudos recentes no pais, onde a gênero feminino comparado ao masculino é identificado com o dobro de transtornos de humor e ansiedade. A depressão como revela o estudo, ultrapassa em muito as outras psicopatologias, no que é seguido por transtornos que tem a ansiedade como queixa e sintoma principal. No que se pode afirmar que $40 \%$ das psicopatologias no serviço de triagem são Episódios Depressivos, e mais de 22\% são de Transtornos Fóbico Ansioso e Outros Transtornos de Ansiedade. O resultado do estudo realizado aponta para a necessidade de projetos de intervenção psicoterápica direcionados a essas psicopatologias e principalmente para as pacientes do gênero feminino usuárias da clínica-escola de psicologia.

\section{REFERÊNCIAS}

AMARAL, A. E. V. et al. Serviços de psicologia em clínicas-escola: revisão de literatura. Bol. psicol. 2012, vol.62, n.136, pp. 37-52. ISSN 0006-5943.

ANDRADE, L. H; VIANA, M. C; SILVEIRA, C. M. Epidemiologia dos transtornos psiquiátricos na mulher. REVISTA DE PSIQUIATRIA CLINICA. 33 (2) 43 - 54, 2006. Disponível em: <http://www.scielo.br/pdf/rpc/v33n2/a03v33n2.pdf>

APOSTOLO, J. L. A, et al. Depressão, ansiedade e estresse em usuários de cuidados primários de saúde. Rev. Latino-Am. Enfermagem. 2011, vol.19, n.2, pp. 348-353.

ARAÚJO, M de F; MATTIOLI, O. C. Gênero e Violência. São Paulo: Artes \& Ciência, 2004. Vários Autores.

BAPTISTA, M. N; BAPTISTA, A. S. B; OLIVEIRA, M. G. Depressão e gênero: por que as mulheres deprimem mais que os homens? 1999, Vol. 7 no 2, pp 143-156.

CAMPEZATTO, P. M.; NUNES, M. L. T. Caracterização da clientela das clínicas-escolas de cursos de Psicologia da região metropolitana de Porto Alegre. Psicologia: Reflexão e Critica 2007, v.20, n.3, pp. 376-388. OK. http://dx.doi.org/10.1590/S0102-79722007000300005

CAMPOS, L. F. DE L. Métodos e Técnicas de Pesquisa em Psicologia. Campinas, SP: Editora Alínea, 2000. 
CASTRO, M. G. T, et al. Relação entre gênero e sintomas depressivos e ansiosos em tabagistas. Revista de psiquiatria. Rio Grande Sul. 2008, vol.30, n.1, pp. 25-30. http://dx.doi.org/10.1590/S0101$\underline{81082008000100008}$

COOPER. J. E. Classificação dos transtornos metais e de comportamento da CID - 10: Referência Rápida/ Organização Mundial de Saúde; Trad. Gabriela Baldisserotto. - Porto Alegre: Artes Médicas, 1997.

CORDIOLI, Aristides Volpato. Psicoterapias: Abordagens atuais. 3.ed. Porto Alegre: Artmed, 2009.

DALGALARRONDO, P. Psicopatologia e semiologia dos transtornos mentais. 2.ed. Porto Alegre: Artmed, 2008.

FURUYA, Rejane Kiyomi et al. Ansiedade e depressão entre homens e mulheres submetidos à intervenção coronária percutânea. Rev. esc. enfermaria. USP. 2013, vol.47, n.6, pp. 1333-1337. ISSN 0080-6234.

JUSTO, L. P; CALIL, H. M. Depressão: o mesmo acometimento para homens e mulheres? Revista de Psiquiatria Clinica. 2006;33:74-9. http://dx.doi.org/10.1590/S0101-60832006000200007

KAPLAN, H. I; SADOCK, B. J; GREBB, J. A. Compêndio de Psiquiatria: Ciências do Comportamento e Psiquiatria Clínica. 7. Ed. Porto Alegre: Artmed, 1997.

KINRYS, G; WYGANT, L. E. Transtornos de ansiedade em mulheres: gênero influencia o tratamento? Rev. Bras. Psiquiatr. 2005, vol.27, suppl.2, pp. s43-s50. http://dx.doi.org/10.1590/S1516$\underline{44462005000600003}$

LAGO, M. C. S. Sobre trabalho, casas, mulheres, ainda. Saberes e Fazeres de Gênero Entre Local e Global. Organizadoras: Luzinette Simões Minella, Susana Bornéo Funck. Florianópolis: Ed. Da UFSC. 2006 pp.245-256.

MARAVIESKI, S.; SERRALTA, F. B. Características clínicas e sociodemográficas da clientela atendida em uma clínica-escola de psicologia. Temas psicol. v.19, n. 2, p. 481-490, dez. 2011

MOLINA, Mariane Ricardo Acosta Lopez et al. Prevalência de depressão em usuários de unidades de atenção primária. Rev. psiquiatr. clín. 2012, vol.39, n.6, pp. 194-197. http://dx.doi.org/10.1590/S0101-60832012000600003

PERFEITO, H. C. C. S.; MELO, S. A. Evolução dos processos de triagem psicológica em uma clínicaescola. Estudos de Psicologia, Campinas, v. 21, n. 1, p. 33-42. 2004. http://dx.doi.org/10.1590/S0103-166X2004000100003

ROCHA, M. Plantão psicológico e triagem: aproximações e distanciamentos. Ver. NUFEN Vol.3 São Paulo, 2011.

ROMARO, R.A; CAPITÃO, C.G. (2003). Caracterização da clientela da clínica-escola de Psicologia da Universidade São Francisco. Psicologia: Teoria e Prática, 5 (1), 111-121. 
ROMARO, R.A. \& OLIVEIRA P.E.C.L. (2008). Identificação das queixas de adultos separados atendidos em uma clínica-escola de Psicologia. Psicologia Ciência e Profissão, 28 (4), 780-793. http://dx.doi.org/10.1590/S1414-98932008000400010

SÁ-SILVA, J R; ALMEIDA, C D de; GUIDANI, J. F. Pesquisa documental: pistas teóricas e metodológicas. Revista Brasileira de História \& Ciências Sociais. Ano I - Número I - julho de 2009.

SILVARES, E. F. M. Atendimento Psicológico em Clínicas-Escola Editora Alínea. Campinas - SP 2006.

SIMÕES, A. et. al. Clínica-Escola de Psicologia: Caracterização do Perfil da Clientela atendida. 2012 [online] Acesso em:10/05/2015. Disponível em: http://aems.edu.br/iniciacaocientifica/download/3ac28c7130.pdf

SIQUEIRA, T. L. Joan Scott e o Papel da História na Relações das Construção de Gênero. Revista Ártemis Vol. 8 junhos 2008, pp 110 - 117. Acesso: 10/06/2015. Disponível em:http://www.pibid.ufpr.br/pibid new/uploads/edfisica2011/arquivo/87/joan scott.pdf

SONENREICH, C; BASSITT, W. O Conceito de Psicopatologia: Distúrbio Psíquico, Doença, Anormalidade. EDITORA MANOLE, 10 Edição, 1979.

TENG, C. T; HUMES, E. DE C; DEMETRIO, F. N. Depressão e comorbidades clínicas. Rev. psiquiatr. clín. 2005, vol.32, n.3, pp. 149-159. ISSN 0101-6083.

VIANA, M. C.; ANDRADE, L H. Lifetime Prevalence, age and gender distribution and age-of-onset of psychiatric disorders in the São Paulo Metropolitan Area, Brazil: results from the São Paulo Megacity Mental Health Survey. Revista Brasileira Psiquiatria 2012, vol.34, n.3. http://dx.doi.org/10.1016/j.rbp.2012.03.001 